\title{
Front Matter: Volume 9655
}

, "Front Matter: Volume 9655," Proc. SPIE 9655, Fifth Asia-Pacific Optical Sensors Conference, 965501 (20 July 2015); doi: 10.1117/12.2203414

EDent: Fifth Asia Pacific Optical Sensors Conference, 2015, Jeju, Korea, SPIE. Republic of 


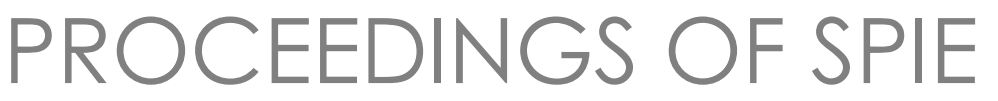

\title{
Fifth Asia-Pacific Optical Sensors Conference
}

\author{
Byoungho Lee \\ Sang Bae Lee \\ Yunjiang Rao \\ Editors
}

20-22 May 2015

Jeju, Korea, Republic of

Organized by

Optical Society of Korea (Korea, Republic of)

Published by

SPIE 
The papers included in this volume were part of the technical conference cited on the cover and title page. Papers were selected and subject to review by the editors and conference program committee. Some conference presentations may not be available for publication. The papers published in these proceedings reflect the work and thoughts of the authors and are published herein as submitted. The publisher is not responsible for the validity of the information or for any outcomes resulting from reliance thereon.

Please use the following format to cite material from this book:

Author(s), "Title of Paper," in Fifth Asia-Pacific Optical Sensors Conference, edited by Byoungho Lee, Sang Bae Lee, Yunjiang Rao, Proceedings of SPIE Vol. 9655 (SPIE, Bellingham, WA, 2015) Article CID Number.

ISSN: 0277-786X

ISBN: 9781628418651

Published by

SPIE

P.O. Box 10, Bellingham, Washington 98227-0010 USA

Telephone +1 3606763290 (Pacific Time) · Fax +1 3606471445

SPIE.org

Copyright @ 2015, Society of Photo-Optical Instrumentation Engineers.

Copying of material in this book for internal or personal use, or for the internal or personal use of specific clients, beyond the fair use provisions granted by the U.S. Copyright Law is authorized by SPIE subject to payment of copying fees. The Transactional Reporting Service base fee for this volume is $\$ 18.00$ per article (or portion thereof), which should be paid directly to the Copyright Clearance Center (CCC), 222 Rosewood Drive, Danvers, MA 01923. Payment may also be made electronically through CCC Online at copyright.com. Other copying for republication, resale, advertising or promotion, or any form of systematic or multiple reproduction of any material in this book is prohibited except with permission in writing from the publisher. The CCC fee code is 0277-786X/15/\$18.00.

Printed in the United States of America.

Publication of record for individual papers is online in the SPIE Digital Library.

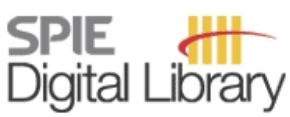

SPIEDigitalLibrary.org

Paper Numbering: Proceedings of SPIE follow an e-First publication model, with papers published first online and then in print. Papers are published as they are submitted and meet publication criteria. A unique citation identifier (CID) number is assigned to each article at the time of the first publication. Utilization of CIDs allows articles to be fully citable as soon as they are published online, and connects the same identifier to all online, print, and electronic versions of the publication. SPIE uses a six-digit CID article numbering system in which:

- The first four digits correspond to the SPIE volume number.

- The last two digits indicate publication order within the volume using a Base 36 numbering

system employing both numerals and letters. These two-number sets start with 00, 01, 02, 03, 04, $05,06,07,08,09,0 A, 0 B \ldots$ OZ, followed by 10-1Z, 20-2Z, etc.

The CID Number appears on each page of the manuscript. The complete citation is used on the first page, and an abbreviated version on subsequent pages. 


\title{
Contents
}

\author{
xiii Authors \\ xix Conference Committee \\ xxiii Introduction \\ xxv Sponsors
}

\section{TOPIC 1 PHYSICAL SENSING}

965502 Multi-channel optical gap sensor based on spectrally resolved interferometry [9655-44]

965503 Imaging spectrophotometer for 2D spatially resolved measurements of spectral reflectance of materials [9655-53]

965504 Distributed feedback fiber laser for sub-nanostrain-resolution static strain measurement by use of swept beat-frequency demodulation [9655-56]

965505 Performance of closed-loop resonant micro-optic gyro with hybrid digital phase modulation [9655-69]

965506 Polarimetric fiber vibration sensor based on polarization-diversity loop structure [9655-74]

965507 Fiber optic Doppler velocimeter based on a dual-polarization fiber grating laser [9655-100]

965508 Optical fiber temperature sensor with $\mathrm{mK}$ resolution and absolute frequency reference [9655-106]

965509 Feature regulation and applications of M-FBG by laser ablation [9655-1 10]

9655 0A High-sensitivity strain sensors based on in-fiber reshaped air bubbles [9655-122]

9655 OB A dual channel method for simultaneous evaluation in two branches of a multi-functional integrated optic chip [9655-123]

9655 OC Optimized Phase Generated Carrier (PGC) demodulation algorithm insensitive to $C$ value [9655-126]

9655 OD Sagnac interferometer based stable phase demodulation system for diaphragm based acoustic sensor [9655-129]

9655 OE Single layered flexible photo-detector based on perylene/graphene composite through printed technology [9655-137]

9655 OF Stretchable photo sensor based on graphene/perylene composite utilizing electrohydrodynamic fabrication technique [9655-140] 
9655 OG High-sensitivity gas pressure sensors based on in-fiber devices [9655-141]

$9655 \mathrm{OH} \quad$ An implementation of non-contact optical fiber displacement sensor using bidirectional modulation of a Mach-Zehnder electro-optical modulator [9655-166]

$9655 \mathrm{Ol} \quad$ Hybrid air-core photonic bandgap fiber ring resonator and implications for resonant fiber optic gyro [9655-181]

9655 0J Research of the differential-type optical fiber F-P vibration sensor with large frequency range [9655-191]

9655 OK Improvement in topology measurement accuracy of atomic force microscope using additional sensor [9655-196]

$9655 \mathrm{OL} \quad$ A fiber optic cure monitoring technique with accuracy improvement of distorted embedded sensors [9655-197]

9655 OM Pressure sensing of Fabry-Perot interferometer with a microchannel demodulated by a FBG [9655-199]

$96550 \mathrm{~N} \quad$ A hybrid fiber optic sensor system for condition monitoring of large scale wind turbine blades [9655-213]

965500 Hetero-core fiber optic tactile sensor for accurate discrimination of texture and hardness [9655-215]

9655 OP SpiderSpec: a low-cost compact colorimeter with loT functionality [9655-230]

$96550 Q$ Dispersion characterization of group birefringence in polarization-maintaining fiber using a Kerr phase-interrogator [9655-233]

9655 OR Sensing properties of periodic stack of nano-films deposited with various vapor-based techniques on optical fiber end-face [9655-238]

9655 OS Simple top down imaging measurement of contact angle for practical assessment of hydrophilic surfaces [9655-258]

9655 OT Optical imaging of air and water bubbles flowing through oil [9655-259]

9655 OU Multipoint vibration sensing using fiber Bragg gratings and optical frequency domain reflectometry [9655-274]

9655 OV Slope-assisted complementary-correlation optical time-domain analysis of Brillouin dynamic gratings for high sensitivity, high spatial resolution, fast and distributed fiber strain sensing [9655-280]

9655 OW Fiber optic flowmeter based on distributed feedback fiber laser [9655-289]

$96550 X \quad$ Fiber Bragg grating ultrasonic sensors based on intensity modulation technique (Invited Paper) [9655-308] 
9655 OY Optical oxygen sensor based on time-resolved fluorescence [9655-4]

$96550 Z$ Methanol selective fibre optic gas sensor with a nanoporous thin film of organic-inorganic hybrid multilayers [9655-46]

965510 A tapered optical fiber interferometer study on the microdynamics phase separation mechanism of the poly( $\mathrm{N}$-isopropylacrylamide) aqueous solution [9655-83]

965511 Fluorescent measurements of $\mathrm{Zn}^{2+}$ on a smartphone [9655-112]

965512 In-line chemical sensor based on C-type fiber and novel photonic crystal fibers with high sensitivity and fast dynamic response [9655-116]

965513 Microfiber Bragg grating hydrogen sensor base on co-sputtered Pd/Ni composite film [9655-119]

965514 Perpendicular coupling glass capillary based interferometric real-time microfluidic sensor [9655-125]

965515 Multi-point hydrogen detection system using hetero-core structured optical fiber hydrogen tip sensor based on surface plasmon resonance and pseudorandom noise code correlation reflectometry [9655-152]

965516 Optical characteristics of pesticides measured by terahertz time domain spectroscopy [9655-157]

965517 A new fiber-tip Fabry-Perot interferometer and its application for pressure measurement [9655-171]

$965518 \quad$ U-shaped evanescent wave optical fibre sensor based on a porphyrin anchored nanoassembled thin film for high sensitivity ammonia detection [9655-203]

965519 Nanocrystalline diamond microelectrode on fused silica optical fibers for electrochemical and optical sensing [9655-239]

96551 A Selective vapor detection of an integrated chemical sensor array [9655-268]

9655 1B Differentiation of vapor mixture with chemical sensor arrays (Invited Paper) [9655-305]

TOPIC 3 BIOLOGICAL/BIOMEDICAL SENSING AND IMAGING

9655 1C A novel single fiber optical tweezers based on light-induced thermal effect [9655-23]

9655 1D A mode-division-multiplexing single fiber optical tweezers [9655-25]

9655 1E Polymer fiber Bragg grating force sensors for minimally invasive surgical devices [9655-67] 
$9655 \mathrm{IF} \quad$ In situ and ultrasensitive DNA detection based on a graphene-coated silica fiber taper interferometer [9655-88]

$96551 G \quad$ Terahertz spectral characteristics of biological tissues [9655-132]

$9655 \mathrm{1H} \quad$ Fabrication and characterization of V-groove liquid core waveguide [9655-153]

$965511 \quad$ Highly sensitive terahertz sensor for glucose detection [9655-156]

$96551 \mathrm{~J}$ Accurate and in situ monitoring of bacterial concentration using a real time all-fibre spectroscopic device [9655-158]

$96551 \mathrm{~K}$ Optical fiber sensing of human skin emanations [9655-179]

9655 1M Holographic 3D fluorescence microscopy (Invited Paper) [9655-235]

9655 IN Orthodontic mechanics using mini-implant measured by FBG [9655-237]

965510 A single-shot 2D/3D simultaneous imaging microscope based on light field microscopy [9655-262]

9655 IP Biomedical sensing and imaging for the anterior segment of the eye (Invited Paper) [9655-296]

$96551 Q \quad$ Surface plasmon scattering: an alternative approach for optical fibers biosensors (Invited Paper) [9655-303]

TOPIC 4 INTEGRATED TECHNOLOGIES FOR SENSING

9655 IR Polarization-sensitive plasmonic hot spot tuning with nanoslit arrays [9655-37]

9655 is Quantum opto-mechanical coupling model for fiber micro-cantilever beam damping noise reduction [9655-48]

9655 IT A novel twin-core fiber connector [9655-51]

$96551 \mathrm{U}$ Side polished twin-core fiber coupler [9655-57]

9655 IV Twin-core fiber end polish technique for particle trapping [9655-61]

9655 IW Ultra-sensitive temperature sensor based on liquid crystal infiltrated photonic crystal fibers [9655-94]

$96551 \mathrm{~A} \quad$ All-fiber Sagnac loop hybrid interferometer based on a highly birefringent photonic crystal fiber with two asymmetric cores and its sensing applications [9655-115]

$96551 Y$ Thermo-optically tunable switching in an electro-microtube ring resonator [9655-135]

965512 Absorption and fluorescence spectroscopy on a smartphone [9655-147] 
965520 Grating decoupler of channel plasmon polariton waveguide for optical intergrated circuit application [9655-164]

965521 High performance refractive index sensing in multimode plasmonic waveguide [9655-165]

965522 Study on the strain characteristic of fiber optic flexural disk accelerometer [9655-175]

965523 Single-shot, long-range, and zoomable optical tomography and profilometry using a diffraction grating and a CCD camera [9655-188]

965524 Modal analysis of rotating plate using tracking laser Doppler vibrometer: algorithm modification [9655-209]

965525 Multi-channel measurement for hetero-core optical fiber sensor by using CMOS camera [9655-216]

965526 Linear polarization sensor with modified Shack-Hartmann wave-front sensor [9655-266]

965527 Microbubble resonators as enhancement plafforms for linear and nonlinear applications (Invited Paper) [9655-298]

\section{TOPIC 5 SPECIALTY OPTICAL FIBERS FOR SENSING}

965528 Design of a high-birefringence two-core photonic crystal fiber for simultaneous measurement of pressure and temperature [9655-8]

965529 Simplified correlation-domain Brillouin sensor using plastic optical fiber [9655-10]

9655 2A Strain characteristics of selectively infiltrated photonic crystal fibers [9655-22]

9655 2B Supercontinuum generation through DNA-filled hollow core fiber for broadband absorption spectroscopy [9655-45]

9655 2C Building a lab-in/on-fiber (Invited Paper) [9655-47]

9655 2D Heterodyning fiber laser based magnetic field sensor using magnetostrictive composite material [9655-78]

9655 2E Preparation of ytterbium-doped silica optical fiber using MCVD process with chelate precursor [9655-91]

$96552 \mathrm{~F}$ Excitation characteristics of seven-core fiber in splicing process [9655-104]

$96552 \mathrm{G}$ Temperature sensitivity of the inline interferometer based on a two-core photonic crystal fiber selectively filled with polymer [9655-117]

$96552 \mathrm{H} \quad$ A high-sensitivity chemical sensor based on titania coated optical-fiber long period grating for ammonia sensing in water [9655-120]

$965521 \quad$ Modal-interference-based temperature sensing using plastic optical fibers: markedly enhanced sensitivity near glass-transition temperature [9655-159] 
$96552 \mathrm{~J} \quad$ Sensing nanometric displacement of a micro-/nano-fiber induced by optical forces by use of white light interferometry [9655-162]

$96552 \mathrm{~K}$ Performance evaluation of an ultra-thin fiber optic dosimeter using therapeutic photon beams [9655-163]

$96552 \mathrm{~L} \quad$ A fiber optic spectrometer produced by a femtosecond 400-nm second harmonic Ti:sapphire laser [9655-194]

$96552 \mathrm{M}$ Self Q-switching of a bismuth doped silica fiber operating at $1.46 \mu \mathrm{m}$ [9655-248]

$96552 \mathrm{~N} \quad$ A microdroplet-etched fiber Fabry-Perot resonator for the refractive index sensing [9655-249]

965520 Improvement of radiation resistance of Er-doped photonic crystal fiber source by spectrum trimming [9655-250]

$96552 \mathrm{P}$ Sensing characteristics of all-solid photonic bandgap fiber modal interferometers [9655-252]

$96552 Q \quad$ Design for an ultra-broad band single-polarization single-mode photonic crystal fiber based on the zero-order Surface Plasmon Polariton mode [9655-256]

$96552 R$ Sensing characteristics of Brillouin scattering spectra in a micro-scaled silica fiber [9655-285]

$96552 S$ Optical Bragg grating sensor fibers for ultra-high temperature applications (Invited Paper) [9655-297]

\section{TOPIC 6 GRATING AND COMPONENT TECHNOLOGIES FOR SENSING}

9655 2T Regenerated long period gratings (LPGs) in boron-codoped germanosilicate optical fibre [9655-11]

$96552 \mathrm{U}$ The characteristic of gap FBG and its application [9655-34]

$96552 \mathrm{~V}$ Sensitive strain sensor based on regenerated microfiber Bragg grating for high temperature environment [9655-39]

9655 2W Pattern matching based smart interrogation algorithm for fiber Bragg gratings inscribed by femtosecond laser [9655-65]

$96552 X \quad$ Beat frequency dependence of the sensitivity for Faraday-rotation based heterodyning fiber laser magnetic field sensor [9655-89]

$96552 Y \quad$ Noise performance improvement of dual-polarization fiber grating laser through external optical feedback [9655-101]

965522 Fabrication of fiber Bragg gratings in embedded-core hollow optical fiber [9655-109] 
965530 Theoretical and experimental study on crosstalk caused by multiple reflections in identical weak fiber Bragg grating array [9655-113]

$965531 \quad$ Planar waveguide Michelson interferometer fabricated by using $157 \mathrm{~nm}$ mask laser micromachining [9655-124]

965532 Tunable plasmonic characteristics at asymmetric double metal caps on a dielectric nanosphere [9655-154]

965533 Chirality measurements using optical fibre long period gratings fabricated in high birefringent fibre [9655-205]

965535 Long period grating sensors response to photosensitive bacteriorhodopsin coating [9655-218]

965536 Novel two-dimensional single-shot optical imaging system by VIPA-comb interferometry [9655-240]

965537 Temperature and strain measurement by using cascaded chirped long period fiber gratings [9655-251]

965538 High-resolution compact dual-frequency fiber laser accelerometer [9655-253]

965539 Combining regenerated gratings and optical fibre Fabry-Pérot cavities for dual sensing of ultra-high temperature and strain [9655-278]

\section{TOPIC 7 NOVEL MATERIALS FOR SENSING}

9655 3A Metal ion sensing solution containing double crossover DNA [9655-160]

$96553 \mathrm{~B} \quad$ Fiber temperature sensor with nanostructured cladding by $\mathrm{TiO}_{2}$ nanoparticles selfassembled onto a side polished optical fiber [9655-169]

$96553 C$ Study on the electrical control of graphene with single-stranded DNA [9655-272]

9655 3D Measurement of mechanoluminescence radiance of $\mathrm{ZnS}: \mathrm{Mn}$ [9655-279]

$96553 \mathrm{E} \quad$ Fabrication of printed ITO sensor for the ammonia hydroxide detection [9655-354]

$96553 \mathrm{~F}$ Recent advances on optical reflectometry for access network diagnostics and distributed sensing [9655-398]

TOPIC 8 DISTRIBUTED, MULTIPLEXED AND NETWORKED SENSING

$96553 G \quad$ Multi optical path generator for fiber optic strain sensors multiplexing [9655-55]

965531 Utilization of negative beat-frequencies for maximizing the update-rate of OFDR [9655-82] 
$96553 \mathrm{~J} \mathrm{High} \mathrm{resolution} \mathrm{optical} \mathrm{time-domain} \mathrm{reflectometry} \mathrm{based} \mathrm{on} \mathrm{correlation} \mathrm{utilizing} \mathrm{an} \mathrm{all-fiber}$ chaotic source [9655-105]

$96553 \mathrm{~K}$ Spectrum zooming in network topology based on a white light fiber optic Mach-Zehnder interferometer [9655-145]

$96553 \mathrm{~L} \quad$ Brillouin optical correlation domain reflectometry with lock-in detection scheme (Invited Paper) [9655-161]

$96553 \mathrm{M}$ A comparative study for massive data compression in long-distance distributed optical fiber sensing systems [9655-204]

$96553 \mathrm{~N} \quad$ Characterization of temperature-dependent birefringence in polarization maintaining fibers based on Brillouin dynamic gratings [9655-231]

96553 P A novel optical coherent domain reflectometer with dual frequency modulation [9655-275]

TOPIC 9 ENVIRONMENTAL AND STRUCTURAL MONITORING

9655 3R High performance fiber optic sensor based on self referenced FBGs and high-speed dualwavelength pulse coding [9655-81]

$965535 \mathrm{Fe}(\mathrm{C})$-coated optical fiber sensors for corrosion alarm monitoring [9655-128]

$96553 \mathrm{~T} \quad$ Fiber optic ultrasonic sensing systems using PS-FBG for damage monitoring in composite materials (Invited Paper) [9655-174]

$96553 \mathrm{U}$ Multi-scale wavelet decomposition and its application in distributed optical fiber fences [9655-176]

9655 3V A fiber optic epoxy cure monitoring technique by using a wavelength-swept laser [9655-208]

9655 3W Ultrahigh-sensitivity temperature sensor based on in-fiber Fabry-Perot interferometer [9655-217]

$96553 \mathrm{X}$ Temperature and strain dependence of the Brillouin frequencies in tapered optical fiber [9655-270]

$96553 Y$ Safety monitoring of the FBG sensor in respect of radioactivity and deformation measurement of a silo structure for radioactive waste disposal [9655-283]

965532 Design and performance analysis of front end optical instrument for coastal water remote sensing [9655-287]

TOPIC 10 INDUSTRIAL APPLICATIONS, FIELD TRIALS AND STANDARDIZATION

965540 Whole optic fiber weighing technique and device of belt conveyor [9655-7] 
965541 Two-phase flow measurement based on oblique laser scattering [9655-80]

965542 Comparisons between co-axial and bi-axial optical systems for time-of-flight based laser scanners [9655-168]

965543 A hydrostatic leak test for water pipeline by using distributed optical fiber vibration sensing system [9655-246]

965544 Multilayered film monitoring using terahertz reflective time-domain spectroscopy [9655-290]

$965545 \quad$ New trends and applications of optical fiber sensing technologies at the NEL-FOST [9655-394]

\section{POST-DEADLINE PAPERS}

965546 In-fiber whispering gallery mode resonator fabricated by femtosecond laser micromaching [9655-400]

965547 High performance distributed acoustic sensor using cyclic pulse coding in a direct detection coherent-OTDR [9655-399]

965548 Long-range and high-resolution correlation optical time-domain reflectometry utilizing an all optical chaotic source [9655-401]

965549 A tunable and switchable wavelength spacing of multi-wavelength erbium doped fiber laser by exploiting nonlinear polarization rotation [9655-402] 
Proc. of SPIE Vol. $9655965501-12$

Downloaded From: https://www.spiedigitallibrary.org/conference-proceedings-of-spie on 26 Apr 2023 Terms of Use: https://www.spiedigitallibrary.org/terms-of-use 


\section{Authors}

Numbers in the index correspond to the last two digits of the six-digit citation identifier (CID) article numbering system used in Proceedings of SPIE. The first four digits reflect the volume number. Base 36 numbering is employed for the last two digits and indicates the order of articles within the volume. Numbers start with 00, 01, 02, 03, 04, 05, 06, 07, 08, 09, OA, OB...0Z, followed by 10-1Z, 20-2Z, etc.

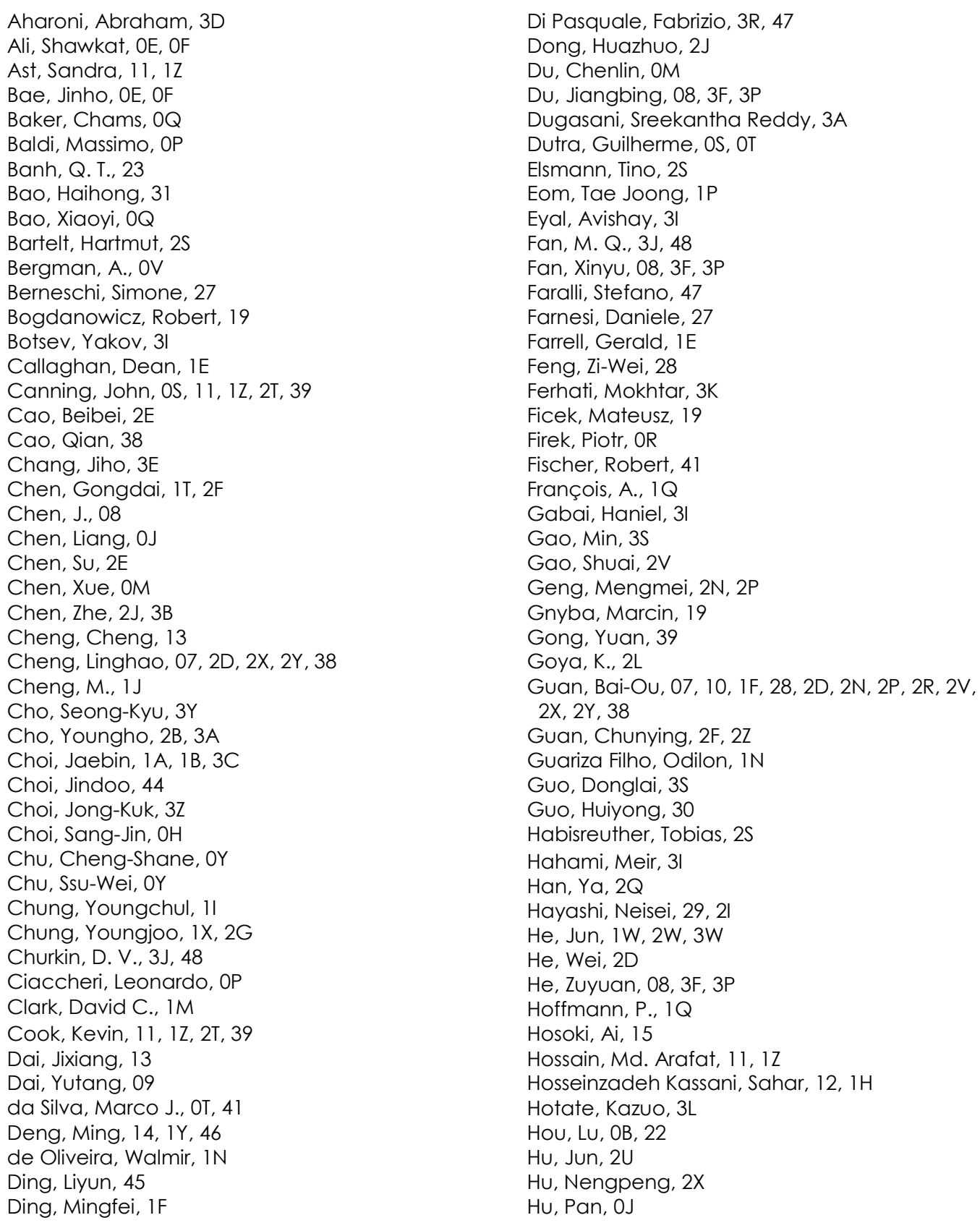

Di Pasquale, Fabrizio, 3R, 47

Dong, Huazhuo, 2J

Du, Chenlin, OM

Du, Jiangbing, 08, 3F, 3P

Dugasani, Sreekantha Reddy, 3A

Dutra, Guilherme, OS, OT

Elsmann, Tino, $2 \mathrm{~S}$

Eom, Tae Joong, $1 \mathrm{P}$

Eyal, Avishay, 3l

Fan, M. Q., 3J, 48

Fan, Xinyu, 08, 3F, 3P

Faralli, Stefano, 47

Farnesi, Daniele, 27

Farrell, Gerald, 1E

Feng, Zi-Wei, 28

Ferhati, Mokhtar, 3K

Ficek, Mateusz, 19

Firek, Piotr, OR

Fischer, Robert, 41

François, A., 1Q

Gabai, Haniel, 31

Gao, Min, 3S

Gao, Shuai, 2V

Geng, Mengmei, 2N, 2P

Gnyba, Marcin, 19

Gong, Yuan, 39

Goya, K., 2L

Guan, Bai-Ou, 07, 10, 1F, 28, 2D, 2N, 2P, 2R, 2V, $2 X, 2 Y, 38$

Guan, Chunying, 2F, $2 Z$

Guariza Filho, Odilon, 1N

Guo, Donglai, 3S

Guo, Huiyong, 30

Habisreuther, Tobias, 25

Hahami, Meir, 3l

Han, Ya, 2Q

Hayashi, Neisei, 29, 2l

He, Jun, 1W, 2W, 3W

He, Wei, 2D

He, Zuyuan, 08, 3F, 3P

Hoffmann, P., $1 Q$

Hosoki, Ai, 15

Hossain, Md. Arafat, 11, 12

Hosseinzadeh Kassani, Sahar, 12, 1H

Hotate, Kazuo, 3L

Hou, LU, OB, 22

$\mathrm{Hu}$, Jun, $2 \mathrm{U}$

Hu, Nengpeng, $2 \mathrm{X}$

$\mathrm{Hu}, \mathrm{Pan}, \mathrm{OJ}$ 
Hu, Peng, 2E

Hu, Qifan, 49

Hu, Wenbin, 35

Huang, Chujia, 45

Huang, Dongmei, 46

Huang, Hankai, 2J

Huang, Jun, 09

Huang, Quandong, OM

Huang, Wei, 46

Huang, Wenzhu, 04, OW

Huang, Yijian, IW

Huang, Yun-Yun, 10, 1F, 2N, 2P, 2V

Hwang, J. H., $1 \mathrm{H}$

Hwang, Sungui, 42

Hyun, Sangwon, $3 z$

Igawa, Hirotaka, 15

Itoh, T., 2L

Jamalipour, Abbas, 11, 1Z

James, S. W., 18, 1K, 2H, 33, 35

Jang, Changwon, $1 \mathrm{M}$

Jang, J. S., 2K

Jang, Junhwan, 42

Jang, K. W., $2 \mathrm{~K}$

Jeon, H., 2K

Jeon, Keum-Soo, $\mathrm{OH}$

Jeong, Jiseong, OK

Jeong, Kyu Hwang, 3X

Jeong, Youngmo, 10

Jhon, Young Miin, 3A

Jiang, Desheng, 30

Jiang, Yuan, 31

Jiang, Zhao-feng, $1 G$

Jin, Long, 2D, 2N, 2V, 38

Jin, Wei, OD, 2U

Jin, Zhonghe, 05, 01

Jo, Songhyun, 06

JoO, B., $1 \mathrm{H}$

Joo, Choun-Ki, IP

Joo, Ki-Nam, 02

Jun, Seong Chan, 1A, 1B, 3A

Jung, Jung-Yeul, 3E

Jung, Minwan, $2 M$

Jung, Soohoon, 3E

Jung, Yongmin, 12

Jung, Youngmo, 1A, 1B, 3C

Kalinowski, Hypolito J., IN

Kang, Chong Yun, 1A, 1B

Kang, Hyukmo, $3 Z$

Karanja, Joseph Muna, 09

Khalil, Hossam, 24

Khazaeinezhad, Reza, 12, 1H

Kim, Beop-Min, 1P

Kim, Bok Hyeon, 1X, 2G

Kim, Bongkyun, 1X, 2G

Kim, Byeong Kwon, 02

Kim, Chulki, 1A, 1B, 2B, 3A, 3C

Kim, Dae-gil, OL, ON, 3V

Kim, Dongkyu, 24

Kim, Geon-Hee, $3 Z$

Kim, Giyoung, 16
Kim, Hwi, 26

Kim, Hyeon-Ho, $\mathrm{OH}$

Kim, Hyo-Suk, 11

Kim, Hyung Min, 2B

Kim, Hyunjin, OL, ON, 3V

Kim, J. S., 2K

Kim, J. S., 2K

Kim, Jae Hun, 1A, 1B, 2B, 3A, 3C

Kim, Jonghyun, $1 \mathrm{M}, 1 \mathrm{O}$

Kim, Joonsoo, 21

Kim, Junsup, OK

Kim, Ki-Soo, 3Y

Kim, Kyuho, 1R

Kim, Kyung-Soo, 44

Kim, Myung K., 1M

Kim, Sang Kyung, 1B

Kim, Soohyun, 44

Kim, Sug-Whan, $3 Z$

Kim, Sun-Je, 1R

Kim, Yong Hyun, 3N

Kim, Young June, 1A, 3C

Kim, Young Suk, 06

Kim, Youngmin, 10

Kishi, Masato, 3L

Klantsataya, E., 1Q

Klinger-Hoffmann, M., $1 Q$

Koba, Marcin, OR

Kobelke, Jens, 12

Koo, Jieun, 3E

Korposh, S., 18, 1K, 2H, 33, 35

Kosakai, T., 23

Koyama, Yuya, 00, 25

Kuang, Zeyuang, 07

Kwon, G., 2K

Kwon, Hyounghan, 20

Kwon, Won Sik, 44

Langer, T., OV

Lao, Yiqin, $2 Y$

Lee, B., 2K

Lee, Byoungho, 1M, 1O, 1R, 20, 21, 26, 32

Lee, Chong Hyun, OE, OF

Lee, Dong-Hoon, 03

Lee, Dong-Kyu, 16, 1

Lee, Hyuk Jae, 2B

Lee, Ji Eun, 1B

Lee, Ju Han, $2 M$

Lee, Kwanil, 2B, 2M, 3X

Lee, Moonjin, 3E

Lee, S.-W., OZ, 18, 1K, 2H, 33

Lee, Sang Bae, $2 M, 3 X$

Lee, Seok, 1A, 1B, 1I, 2B, 3A, 3C

Lee, Seok-hwan, 3E

Lee, Seung-Yeol, 1R, 21

Lee, Taikjin, 1A, 1B, 2B, 3A, 3C

Lee, Yohan, 26

Lee, Yong Wook, 06

Lee, Yong-Eun, IP

Li, Chuang, OB, 22

Li, Fang, 04, OW

$\mathrm{Li}$, Feng, OW 
Li, Haibao, 15

$\mathrm{Li}$, Hanyu, 3M, 3U

Li, Jie, 10, 2N, 2P, 2R, 2V

Li, Song, 3K

Li, Wei, $2 Q$

Li, Weilai, 40

Li, Xiaofu, 30

Li, Y., 3J, 48

Li, Zhengyong, OA, OG, 3W

Liang, Hao, 07, 2R, 2X, $2 Y$

Liang, Peibo, $1 \mathrm{C}$

Liang, S., OC

Liang, Yizhi, 07, 2D, 2X, 2Y, 38

Liao, Changrui, OA, OG, 17, 1W, 3W

Liao, Guozhen, 3B

Lim, Chaehyun, 1A, 1B, 3C

Liv, Bin, 09

Liu, Bo, 2N, 2P

Liv, Chengxiang, 20

Liu, Deming, $2 Q$

Liu, Jie, 40

Liu, Jun, $3 M$

Liu, L., 3P

Liv, Min, 46

Liu, Qingwen, 08, 3F, 3P

Liu, Shen, 0A, 0G, 17, 1W, 3W

Liu, Wei, $1 G$

Liu, Wen, 2T

Liu, Xinrui, OJ

Liu, Xuejing, $2 \mathrm{U}$

Liv, Yang, $2 \mathrm{~F}$

Liu, Zhihai, OB, 1C, 1D

Lopes, Stephani C. P. S., IN

Lu, Cheng-zhen, $1 G$

Lu, Huihui, 2J, 3B

LU, Yang, $O Q$

Luo, Cheng, 30

Luo, Hao, 3G

Luo, Yunhan, 3B

Lydiard, S., $1 \mathrm{~J}$

Ma, Huilian, 05, OI

Ma, Jun, OD

Ma, L., 08, 3P

Mahmoud, Khaled, 03

Mao, Guopei, $2 Z$

Martelli, Cícero, OS, OT, 41

Mathews, Sunish, $1 E$

McGoverin, C., 1J

Mencaglia, Andrea Azelio, OP

Mignani, Anna Grazia, OP

Milczewski, Maura S., IN

Miyaoka, Takumi, 36

Mizuno, Yosuke, 29, 21

Monro, T. M., $1 Q$

Moon, Hi Gyu, 1A, 1B

Morales, Rigoberto E. M., OT, 41

Muanenda, Yonas, 47

Mun, Sang-Eun, 20

Naeem, Khurram, 1X, 2G

Nakamura, Kentaro, 29, 2
Nam, Joonsik, 24

Nannipieri, Tiziano, 3R

Nazari, T., $1 \mathrm{H}$

Ngo, Thanh Tung, 37

Nishiyama, Michiko, 00, 15, 25

Numata, Goki, 21

Nunzi Conti, Gualtiero, 27

Oh, Eunsong, $3 Z$

Oh, Juyeong, 2B, 3A

Oh, Kyunghwan, 12, 1H, 2B

Okabe, Yoji, 3T

Okuda, H., 0Z, 18

Oton, Claudio J., 47

Pan, Jae-Kyung, $\mathrm{OH}$

Pan, Jianjun, 40

Park, Byeongho, 2B, 3A

Park, Hyeonsoo, 20, 21, 32

Park, J., $1 \mathrm{H}$

Park, Kyihwan, OK, 24, 42

Park, Kyoungsoo, 06

Park, Seongchong, 03

Park, Seung-Nam, 03

Park, Sung $\mathrm{Ha}, 2 \mathrm{~B}, 3 \mathrm{~A}$

Park, YoungJe, $3 Z$

Partridge, M., 35

Patyk, Rodolfo L., OT

Paulson, B., $1 \mathrm{H}$

Peng, Feng, OB, 22

Peng, Gang-Ding, $1 \mathrm{E}$

Persits, Nili, 3D

Qian, X. Y., 3J, 48

Qian, Ya, 3U, 43

Qiu, Weiqia, 2J

Quercioli, Franco, 27

Rajan, Ginu, $1 \mathrm{E}$

Ran, Yang, 1F, 2P, 2V

Ran, Zengling, 31, 39

Rao, Yunjiang, 31, 39, 3J, 3M, 3U, 43, 48

Righini, Giancarlo C., 27

Rodrigues, Rômulo L. P., 41

Rothhardt, Manfred, 25

Różycki-Bakon, Radosław, OR

Ruan, Shuangchen, OM, 20

Rutledge, Peter J., 11, 12

Ryl, Jacek, 19

Sampath, Umesh, OL, ON, 3V

Schuster, Kay, $2 S$

Sciacca, B., 1Q

Seki, Atsushi, 15, 2L

Selyanchyn, R., $1 \mathrm{~K}$

Seo, Min Ah, 1A, 1B, 11, 2B, 3A, 3C

Seo, Yong Bum, 02

Shi, Jinhui, $2 Z$

Shi, Leilei, 14, 46

Shin, Beom Ju, 1 A

Shin, Bum JU, 1B

Shin, S. H., 2K

Shioda, Tatsutoshi, 23, 36

Sima, Chaotan, $2 \mathrm{Q}$

Singhal, N., 1J 
Siuzdak, Katarzyna, 19

Śmietana, Mateusz, OR, 19

Sobaszek, Michał, 19

Son, Joo-Hiuk, 16

Song, Eui-Young, 20

Song, Kwang Yong, 3N, $3 \mathrm{X}$

Song, Minho, OL, ON, 3V

Song, Y., $1 \mathrm{~J}$

Soria, Silvia, 27

Sun, Bing, OG, 1W, 3W

Sun, Bo, 2F, $2 Z$

Sun, Cheng, $2 \mathrm{E}$

Sun, Li-Peng, 2N, 2P, 2R, 2V

Sun, Ping, $1 \mathrm{G}$

Sun, Qi, 2R

Sun, Qizhen, 2Q

Sun, Zhenshi, 43

Suwal, O. K., $1 \mathrm{H}$

Swift, S., $1 \mathrm{~J}$

Tabaru, Marie, 2l

Takahashi, Nobuaki, OU, 37

Tam, Hwa-Yaw, 28

Tanaka, Satoshi, OU, OX, 37

Tang, Jieyuan, 3B

Tao, W., $1 \mathrm{~J}$

Tatam, R. P., 2H, 33, 35

Tian, Jie, 2A

Tian, Zhengwen, 3B

Tian, Zhuang, 10

Tiwari, D., 2H

Tong, Xinglin, OJ

Trannin, Pamela G., $1 \mathrm{~N}$

Tsukida, Osamu, 37

Tur, Moshe, OV, 3D

Uchimura, Ryoutarou, 37

Vanholsbeeck, F., 1J

Vendruscolo, Tiago P., OT, 41

Wada, Atsushi, OU, 37

Wang, Chao, IW

Wang, Gaopeng, 13

Wang, Guanjun, 17

Wang, Kun, OJ

Wang, Linglan, 05, 0

Wang, Qiaoni, 2W

Wang, T., OZ, 18, $1 \mathrm{~K}$

Wang, Xianbin, $1 \mathrm{U}$

Wang, Ying, 2A, 3W

Wang, Yiping, OA, OG, 17, 1W, 2W, 3W

Wang, Yiting, 3B

Wang, Z. N., 3J, 48

Watanabe, Kazuhiro, 00, 15, 25, $2 \mathrm{~L}$

Wen, Xiaoyan, 09

Won, Bumsik, 42

Woo, Deok Ha, 2B, 3A

Wu, Bing, OB, OC, 22

Wu, Chuang, 28

Wu, H., 3J

Wu, H., 48

Wu, Huijuan, 3M, 3U, 43

Wu, Qi, 3T
Wu, Xu, 20

Wu, Xuezhong, 31

Xia, Li, 2Q

$X \cup$, Hongbin, $O W$

$X \cup$, Jiwei, $3 M$

$X u$, Xizhen, 17

$\mathrm{Xu}$, Yihang, 2F

Yamazaki, Hiroshi, 00

Yan, Dekai, OB

Yan, Yuchao, 0

Yang, Bing, 3B

Yang, Jing, 2F

Yang, Jun, OB, OC, 1C, 1D, 1V, 22

Yang, Kaiming, OA, 3W

Yang, Ke, 31

Yang, Minghong, 13, 3S, 45

Yang, Yuanhong, 2U, 2W

Yang, Yucheng, 2E

Yang, Zhiming, 14

Yao, Yuguo, 3L

Yeom, Dong-II, 2B

Yi, Jong Chang, $3 C$

Yin, Guolu, OG, 1W, 3W

Ying, Diqing, $\mathrm{Ol}$

Yoo, W. J., 2K

Yoo, Young-Sik, IP

Yoon, Yeomin, OK

Yu, Bo, $1 \mathrm{~F}$

Yu, Haihu, 2E, 30

YU, Jianhui, 2J, 3B

Yu, Kuanglu, $2 Y$

YU, Li, 2X

Yu, WenBing, 2A

YU, Yongqin, OD, OM

$Y U$, Zhangjun, $O B$

Yuan, Li-Bo, OB, OC, 1C, 1D, 1S, 1T, 1U, 1V, 22, 2C,

$2 F, 2 Z, 3 G, 3 K$

Yuan, Qiang, 2D

Yuan, Tingting, 1V, $2 Z$

Yuan, Yinquan, 13, 45

Yuan, Yonggui, OB, OC, 1V, 22, 3G

Yun, Hansik, 32

Zaidi, Farhan, 3R

Zeng, Jing, $1 Y$

Zhang, Jianjie, 05

Zhang, Jun, 3B

Zhang, L., 3J

Zhang, Li, 20

Zhang, Linqiang, 3M, 3U, 48

Zhang, Min, 49

Zhang, Tao, 43

Zhang, Weili, 3M, 3U

Zhang, Wentao, 04, OW

Zhang, Xiaotong, IV

Zhang, Yating, $2 Q$

Zhang, Yaxun, 1C, 1D

Zhang, YU, OB, 1C, ID

Zhao, Enming, 1C, 1D

Zhao, Jing, 17, 1W

Zhao, Minli, OJ 
Zheng, Wanjun, 49

Zheng, Xing, 3S

Zheng, Yu, 30

Zhong, Xiaoyong, 0G, 3W

Zhong, Xing, $2 Z$

Zhou, Chaoran, $0 \mathrm{~J}$

Zhou, Ciming, 45

Zhou, Jiangtao, $1 \mathrm{~W}$

Zhou, Xian, 09

Zhu, Cheng, 3S

Zhu, Jianhui, 20

Zhu, Tao, 14, 1Y, 46

Zuber, A., 1Q

Proc. of SPIE Vol. $9655965501-17$

Downloaded From: https://www.spiedigitallibrary.org/conference-proceedings-of-spie on 26 Apr 2023 Terms of Use: https://www.spiedigitallibrary.org/terms-of-use 
Proc. of SPIE Vol. $9655965501-18$

Downloaded From: https://www.spiedigitallibrary.org/conference-proceedings-of-spie on 26 Apr 2023 Terms of Use: https://www.spiedigitallibrary.org/terms-of-use 


\title{
Conference Committee
}

\author{
Conference Chairs
}

Byoungho Lee, Seoul National University (Korea, Republic of)

Sang Bae Lee, Korea Institute of Science and Technology

(Korea, Republic of)

Yunjiang Rao, University of Electronic Science and Technology of

China (China)

\section{Organizing Committee Members}

Jinho Bae, Jeju National University (Korea, Republic of)

Young-Geun Han, Hanyang University (Korea, Republic of)

Min Yong Jeon, Chungnam National University (Korea, Republic of)

Jaehoon Jung, Dankook University (Korea, Republic of)

Chang-Seok Kim, Pusan National University (Korea, Republic of)

Donghyun Kim, Yonsei University (Korea, Republic of)

Jeehyun Kim, Kyungpook National University (Korea, Republic of)

Yong Wook Lee, Pukyong National University (Korea, Republic of)

Kyunghwan Oh, Yonsei University (Korea, Republic of)

Kwang Yong Song, Chung-Ang University (Korea, Republic of)

Minho Song, Chonbuk National University (Korea, Republic of)

Technical Program Committee Chairs

Youngjoo Chung, Gwangju Institute of Science and Technology,

(Korea, Republic of)

Wei Jin, The Hong Kong Polytechnic University (Hong Kong, China)

Seok Lee, Korea Institute of Science and Technology

(Korea, Republic of)

Kentaro Nakamura, Tokyo Institute of Technology (Japan)

Technical Program Committee Members

Jacques Albert, Carleton University (Canada)

Kevin Chen, Pittsburgh University (United States)

Zhe Chen, Jinan University (China)

Kin Seng Chiang, City University of Hong Kong (Hong Kong, China)

Kevin Cook, The University of Sydney (Australia)

Fabrizio Di Pasquale, Scuola Superiore Sant'Anna (Italy)

Xinyong Dong China Jiliang University (China)

Wolfgang Ecke, Institute of Photonics (Germany)

Yasufumi Enami, Hiroshima University (Japan)

Jayantha Epaarachchi, University of Southern Queensland (Australia)

Brant Gibson, University of Melbourne (Australia) 
Min Gu, Swinburne University of Technology (Australia)

Young-Geun Han, Hanyang University (Korea, Republic of)

Sheng-Lung Huang, National Taiwan University (Taiwan)

Muhammad Khawar Islam, University of Engineering \& Technology

Taxila (Pakistan)

Shibin Jiang, AdValue Photonics Inc. (United States)

Irina Kabakova, The University of Sydney (Australia)

Kyriacos Kalli, Cyprus University of Technology (Cyprus)

Myeong Soo Kang, Korea Advanced Institute of Science and Technology (Korea, Republic of)

Jim Katsifolis, Future Fibre Technologies (Australia)

Chang-Seok Kim, Pusan National University (Korea, Republic of)

II-Bum Kwon, Korea Research Institute of Standards and Science (Korea, Republic of)

Byeong Ha Lee, Gwangju Institue of Science and Technology

(Korea, Republic of)

Ju Han Lee, University of Seoul (Korea, Republic of)

Ming-jun Li, Corning Incorporated (United States)

Seon Do Lim, KRISS (Korea, Republic of)

Tiegen Liu, Tianjin University (China)

Cicero Martelli, Federal University of Technology (Brazil)

Anna Mignani, CNR-Institute of Applied Physics 'Nello Carrara' (Italy)

Arnan Mitchell, RMIT University (Australia)

David Moss, The University of Sydney (Australia)

Koji Omichi, Fujikura Ltd. (Japan)

Luca Palmieri, University of Padova (Italy)

Jaehee Park, Keimyung University (Korea, Republic of)

Gang-Ding Peng, University of New South Wales (Australia)

Halina Rubinsztein-Dunlop, University of Queensland (Australia)

Minah Seo, Korea Institute of Science and Technology,

(Korea, Republic of)

Tatsutoshi Shioda, Saitama University (Japan)

Ki-Bong Song, Electronics and Telecommunications Research Institute (Korea, Republic of)

Kwang Yong Song, Chung-Ang University (Korea, Republic of)

Minho Song, Chonbuk National University (Korea, Republic of)

Sarun Sumriddetchkajorn, National Electronics and Computer Technology Centre (Thailand)

Satoshi Tanaka, National Defense Academy of Japan (Japan)

Graham Town, Macquarie University (Australia)

Dongning Wang, The Hong Kong Polytechnic University

(Hong Kong, China)

Yiping Wang, Shenzhen University (China)

Lei Xu, Fudan University (China)

Changyuan Yu, National University of Singapore (Singapore)

Libo Yuan, Harbin Engineering University (China) 
Xuping Zhang, Nanjing University (China)

Tao Zhu, Chongqing University (China)

International Steering Committee Members

Xiaoyi Bao, University of Ottawa (Canada)

Hartmut Bartelt, Institute of Photonics (Germany)

Arthur Braga, Pontifica Universidade Catolica do Rio de Janeiro (Brazil)

John Canning, The University of Sydney (Australia)

Andrea Cusano, Universita degli Studi del Sannio (Italy)

Bai-Ou Guan, Jinan University (China)

Kazuo Hotate, The University of Tokyo (Japan)

Wei Jin, The Hong Kong Polytechnic University (Hong Kong, China)

Byoung Yoon Kim, Korea Advanced Institute of Science and

Technology (Korea, Republic of)

Yanbiao Liao, Tsinghua University (China)

Byoungho Lee, Seoul National University (Korea, Republic of)

Yu-Lung Lo, National Cheng Kung University (Taiwan)

Alexis Mendez, MCH Engineering (United States)

Kyunghwan Oh, Yonsei University (Korea, Republic of)

Yunjiang Rao, University of Electronic Science and Technology of

China (China)

David D. Sampson, The University of Western Australia (Australia)

Jose Luis Santos, Universidade do Porto (Portugal)

Perry Shum, Nanyang Technological University (Singapore)

Ralph P. Tatam, Cranfield University (United Kingdom)

Hwayaw Tam, The Hong Kong Polytechnic University

(Hong Kong, China)

Frederique Vanholsbeeck, University of Auckland (New Zealand)

Anbo Wang, Virginia Polytechnic Institute and State University

(United States)

Reinhardt Willsch, Institute of Photonics (Germany)

Minghong Yang, Wuhan University of Technology (China) 
Proc. of SPIE Vol. $9655965501-22$

Downloaded From: https://www.spiedigitallibrary.org/conference-proceedings-of-spie on 26 Apr 2023 Terms of Use: https://www.spiedigitallibrary.org/terms-of-use 


\section{Introduction}

The Fifth Asia-Pacific Optical Sensors Conference (APOS 2015) was held 20-22 May 2015, at the Lotte City Hotel Jeju in Jeju, Korea.

APOS 2015 continues a series of conferences that are intended to provide a central forum for an update and review of technical information covering a wide range of optical sensing fields-from fundamental research to systems and applications. The conference is open to researchers and professionals from the Asia-Pacific Rim as well as all regions of the world.

Since the first event held in Chengdu, China in 2008, APOS has become the key regional forum in this field and where an international collection of researchers and industry colleagues could get together to discuss and develop mutual collaborations. It is also the place where we can train the next generation of young researchers and leaders.

APOS 2015 was initiated to provide an open forum discussing state-of-the-art scientific and technological achievements in optical sensors, leading to future industrial applications. The conference included the following topics: Physical Sensing; Chemical and Gas Sensing; Biological/Biomedical Sensing and Imaging; Integrated Technologies for Sensing; Specialty Optical Fibers for Sensing; Grating and Component Technologies for Sensing; Novel Materials for Sensing, Distributed, Multiplexed and Networked Sensing; Environmental and Structural Monitoring; and Industrial Applications, Field Trials and Standardization. Many of the submitted papers described recently developed optical sensors. Sharing the research achievements from this conference will benefit the international scientific community.

This volume contains reviewed papers presented at APOS 2015; a total of 151 papers were selected among 181 submitted abstracts, including three plenary talks, 78 oral presentations, and 100 poster presentations covering 18 sessions. This work was supported by the Korean Federation of Science and Technology Societies Grant funded by the Korean government.

The committee chairs would like to express their sincere thanks to the organizing committee members, program committee members, international steering committee members and also all sponsoring and supporting organizations that offered essential support to the success of APOS 2015. 
Proc. of SPIE Vol. $9655965501-24$

Downloaded From: https://www.spiedigitallibrary.org/conference-proceedings-of-spie on 26 Apr 2023 Terms of Use: https://www.spiedigitallibrary.org/terms-of-use 


\title{
Conference Sponsorship and Support
}

\author{
Conference Organizers \\ Optical Society of Korea \\ Technical Cosponsors \\ SPIE \\ The Optical Society \\ IEEE Photonics Society Center for Relativistic Laser Science \\ Supported by \\ Korea Federation of Science and Technology Societies (KOFST) \\ National Creative Research Center for Active Plasmonics Application \\ Systems (SNU) \\ Korea Institute of Science and Technology (KIST) \\ Korea Tourism Organization \\ Jeju Tourism Organization \\ Jeju Convention \& Visitors Bureau \\ Sponsors \\ FIBERPRO, Inc. \\ SeongKyeong Photonics \\ JT, Inc. \\ Taihan Fiberoptics Co., Ltd. \\ NineOne Co., Ltd. \\ CESCO Co., Ltd. \\ Advanced Photonics Research Institute
}


Proc. of SPIE Vol. $9655965501-26$

Downloaded From: https://www.spiedigitallibrary.org/conference-proceedings-of-spie on 26 Apr 2023 Terms of Use: https://www.spiedigitallibrary.org/terms-of-use 